\title{
Application of Multiobjective Evolutionary Techniques for Robust Portfolio Optimization
}

\author{
Sandra Garcia Rodriguez \\ Complutense University of Madrid, Spain
}

\begin{abstract}
On December 20 of 2012 Sandra García Rodríguez defended his PhD at Carlos III of Madrid (Spain), called: "Application of Multiobjective Techniques for Robust Portfolio Optimization". This thesis was supervised by Dr. David Quintana Montero and Dr. Inés M. Galván León. The defense was done in a publicly open presentation held at Carlos III University of Madrid. The PhD was approved, with the highest rating Cum Laude, by the examining committee: Dr. José Manuel Molina López, Dr. Antonio Gaspar Lopes da Cunha and Dr. David Camacho Fernández.
\end{abstract}

Kewwords - Financial Robust Portfolio Optimization, Multiobjective Evolutionary Algorithms, Artificial Intelligence.

\section{ABSTRACT}

$\mathrm{T}$ he choice of the right way to distribute investor's wealth among different investment alternatives is one of the basic problems that investors must face. Each of these possible combinations, known as financial portfolios, have some characteristics that make them more or less desirable to the investor depending on his preferences. Therefore, this is the reason why the problem of determining the best proportion of capital to assign to each investment asset, the portfolio optimization, has been one of the core topics in financial management research. Academic literature on this subject is very large and mostly based on the seminal work of $\mathrm{H}$. Markowitz [1], who suggests the evaluation of portfolios by computing their associated return and risk.

Hence, the mentioned problem can be considered as a multiobjective optimization problem where the goal is both maximizing return and minimizing risk of the portfolio at the same time. The opposing nature of these objectives provokes that maximizing one of them increments the other too, and viceversa. Thereby, the solution does not consist of a single asset allocation, but a range of them. These portfolios are the ones with the best risk/return trade-off found and define the Efficient Frontier. Thus, this set will be used by the decision maker to choose the portfolio that suits him better according to his preferences. Moreover, when this problem is studied within the real financial world, some constraints should be considered that cannot be hold by traditional optimization methods. This is the reason why we use evolutionary computation as it provides the flexibility necessary to deal with real-world scenarios.

One of the major reasons why some practitioners mistrust quantitative methods based on modern portfolio theory is the lack of robustness that show their solutions. Unfortunately, these portfolios may be very dependant on the estimates of parameters that are unlikely accurate due to, for instance, the presence of outliers. When it is the case, portfolios may behave in a unexpected way driving to wrong investment decisions.

For this reason, we face the robust portfolio optimization problem using four evolutionary multiobjective algorithms (MOEAs) [2]. Moreover, we study three approaches that, adapted to MOEAs, are able to generate portfolios with more stability than the ones provided by standard versions or the "Random Matrix Approach" (RMA) proposed by Plerou et al [3]. The first one includes an explicit objective to measure robustness. The second technique handles robustness implicitly evaluating risk and return of new portfolios under different parameters each generation [4]. The last one improves the previous approach adding a time-stamping mechanism to encourage stable individuals [5].

Finally, since our aim is to deal with the lack robustness, we suggested five new metrics that were used to evaluate the robustness of results. Their analysis showed that the three designed approaches achieved robustness improvements from the $9 \%$ to the $67.5 \%$ over the RMA or the bi-objective basic formulation of the problem. All the observed differences within the three core and RMA algorithms were statistically significant at the $11 \%$ conventional level. There was just one exception that was significant at $5 \backslash \%$. Hence, we expect that this increase in reliability will help portfolio managers to feel more confident about automatic methods for portfolio optimization.

\section{REFERENCES}

[1] Markowitz, H., 1952. Portfolio selection. The Journal of Finance 7 (1), 77-91.

[2] P. Seoane, M. Gestal and J. Dorado. Approach for solving multimodal problems using Genetic Algorithms with Grouped into Species optimized with Predator - Prey. International Journal of Interactive Multimedia and Artificial Intelligence. 1(5), 6-13, 2012.

[3] V. Plerou, P. Gopikrishnan, B. Rosenow, L.A.N. Amaral, T. Guhr and H.E. Stanley: 2002, A random matrix approach to cross-correlations in nancial data, Quantitative finance papers.

[4] García Sandra, Quintana David, Galván Inés M., and Isasi Pedro. Multiobjective Algorithms with Resampling for Portfolio Optimization. Computing and informatics. In Press, Corrected Proof. 
[5] García Sandra, Quintana David, Galván Inés M., and Isasi Pedro. Timestamped Resampling for Robust Evolutionary Portfolio Optimization. Expert Systems with Applications. 39(12), 10722-10730. 2012.
Sandra Garcia Rodriguez graduated in Computer Science Engineering at Carlos III University of Madrid. After the five-year engineering she studied a specialization master on Artificial Intelligence and then the $\mathrm{PhD}$ in the same area. From Sept, 2007 to April, 2013 she worked at the Department of Computer Science, University Carlos III of Madrid, as a professor and researcher participating in several international projects. Since May,2013 she has been working in the ISIA department at Complutense University of Madrid. Her current research focuses in artificial neural networks, evolutionary computation techniques (as genetic algorithms, evolutionary strategies, and particle swarm), multi-objective optimization and robust portfolio optimization. 